\title{
Face recognition based on regularized Gabor fusion contour wave two transform
}

\author{
Yingjie Zhao, Yisu Liu \\ 1 Northeastern University at Qinhuangdao Control engineering Hebei Province Qinhuangdao City \\ 066004
}

627392465@qq.com

Key words: face recognition; occlusion; contourlet; quadratic transformation; feature selection

\begin{abstract}
This paper has, from the aforementioned two perspectives, put forward an algorithm for face recognition through a combined use of local descriptor of geometrical information and neighborhood information on the surface of face. Method: firstly, extract a contour line with a geodesic distance equal to that from the pronasale on the face after posture correction; then extract the local neighborhood information of each point in the re-sampled contour line to constitute the local descriptor; finally, carry out the weighted summation rule fusion of local descriptor between corresponding points of the tested face and collected face, and then use the nearest neighbor classifier to obtain the final recognition results. It indicates that this algorithm, whether in the recognition rate or in the computation complexity, can achieve relatively good effect.
\end{abstract}

\section{Introduction}

As an important biometric feature recognition method, face recognition is characterized by low interference and high chance of being accepted, with broad application prospects in biometric authentication. Three-dimensional (3D) face recognition is carried out based on the 3D geometric shape information of the face, and it is not subject to the impact of posture and light; and with rapid development of 3D scanning technology, 3D face recognition has received more and more attention $[1,2]$. There are, at present, numerous researches on 3D face recognition methods, which are mainly divided into the method based on overall features and the method based on local features. However, the method based on overall features is easily affected by changes in posture, and it is not able to well deal with the impact by occlusion of hair and beard, etc. With regard to the method based on local features, face recognition is achieved by recognizing features of partial areas on the face. Such method, which can achieve different treatment for different areas of the face in accordance with the corresponding features, has a certain advantage in dealing with the problem of facial expression and occlusion over the method based on overall features. Various local features can be used for 3D face recognition, and the most common are the features based on geometrical information and neighborhood information on surface of the face. Pan et al [3] put forward a mirror model matching method to detect the symmetry plane of 3D face, thus obtaining side contour lines of the face center; after alignment of the contour lines, the improved Hausdorff distance can be used to measure the similarity degree of contour lines and complete the face recognition. Drira et al [4], taking pronasale as the center, extracted the radial silhouette line on surface of the face, and then calculated the similarity degree of two faces by using the Elastic Graph Matching technology (all these were carried out based on geometrical information on surface of the face). Wang et al [5] came up with the 3D face recognition method based on Spin Sphere Image (SSI), in which the shape information of neighborhood surface in an arbitrary point of the face is presented in the form of column diagrams. Al-Osaimi et al extracted the overall and local 0-order tensor of any point on the surface as the features to carry out facial shape recognition (all these were carried out based on neighborhood information of an arbitrary point on the surface). 


\section{Pre-processing and contour line extraction}

Face data pre-processing

Face data obtained through 3D scanner is, in general, under different postures, and may involve different areas of the face, including ears, hair and neck, etc. To accurately extract the features and improve the accuracy of follow-up matching, a certain extent of cutting should be carried out on the face; then corresponding posture correction should be implemented to obtain relatively accurate face area.

In this paper, reference to literature [1] is adopted to achieve normalization of face posture. Method: firstly, determine the position of pronasale based on the face shape index characteristics and geometric constraints; then draw a sphere (radius: $r$ ) with pronasale as the center and define the area within the sphere as the final face area. 3D face point cloud data can indicate actual size of the face. Face area is, in general, centralized within a sphere with pronasale as the center and a radius of $90 \mathrm{~mm}$; so a face cutting shall be carried out based on a radius of $90 \mathrm{~mm}$, namely $r=90 \mathrm{~mm}$. Original face and post-cutting face are shown in Fig. 1 (a) and (b). Carry out the PCA (Principal component analysis) on point cloud of the post-cutting face, three mutually perpendicular main directions can be obtained; build a coordinate system with the three main directions, and in such coordinate system, the face should have the same positive posture. Such coordinate system is also named as PCS (Pose Coordinate System). Take the pronasale as starting point of PCS and then convert all point cloud data of the post-cutting face into PCS, thus achieving the face posture correction, as is shown in Fig. 1 (c).
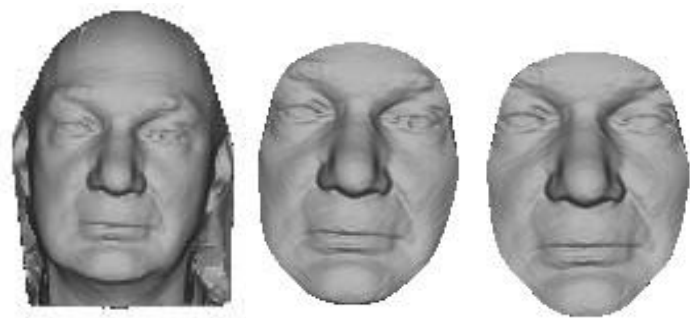

(a) Original face; (b) Post-cutting face; (c) Face in the Pose Coordinate System (PCS)

Fig. 1 Face Cutting and Posture Correction

Extraction of equal-geodesic contour lines

The shortest distance between two points on the surface is taken as the geodesic distance, which is considered to be free from impact of changes in the face expressions [9]. In this paper, the method put forward by Dijkstra et al [10] was used to calculate the geodesic distance between two arbitrary points in point cloud of the face.

After calculating the geodesic distance between the pronasale and all points of the face, the points, geodesic distance of which from the pronasale is within the range $\mathrm{e}^{[\gamma-\delta, \gamma+\delta]}$, shall be selected to constitute equal-geodesic contour lines $c^{\prime}$. In fact, $c^{\prime}$ is a closed band region constituted by a series of points, and different $\delta(\delta>0)$ can change width of such band region (in this paper, $\delta=1.4 \mathrm{~mm})$. Different $\gamma$ can help obtain different equal-geodesic contour lines, and number/location of equal-geodesic contour lines shall make a big difference to representation of contour lines. To better recognize the face shape, the value of $\gamma$ shall be defined as $\gamma=\{5,10,15,20,25,30,35,40,45,50,55,60,65,70,75,80,85\}$, and 17 equal-geodesic contour lines $c_{i}{ }^{\prime}(i=1,2, \ldots, 17)$ shall be extracted within the face area at equal interval; such 17 contour lines basically cover the entire face area, as is shown in Fig. 2. 

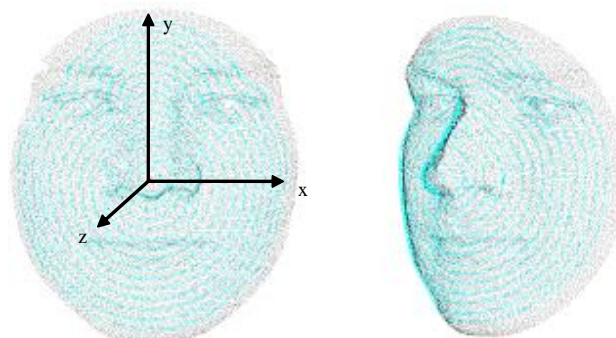

(a) Front view; (b) Side view

Fig. 2 Equal-geodesic contour lines

Sampling of equal-geodesic contour lines

Number of equal-geodesic contour lines extracted from different faces based on the above-mentioned method is different in general, and a comparison in features of corresponding points in different line shall be carried out during recognition. Hence, re-sampling of equal-geodesic contour lines is required before carrying out the feature matching.

It can be found from Fig. 3 that the projection of equal-geodesic contour lines on the principal axis coordinate system ${ }^{x O y}$ is approximate to an ellipse. if the pronasale is taken as the center, projection difference value (a) of equal-geodesic contour lines on $\mathrm{x}$-axis is taken as the minor axis and projection difference value (b) of equal-geodesic contour lines on y-axis is taken as the major axis, an ellipse can be obtained, and its equation is shown as follow:

$$
\left\{\begin{array}{l}
x=\frac{a}{2} \cos (t) \\
y=\frac{b}{2} \sin (t)
\end{array}\right.
$$

Where, ${ }^{t=[0,2 \pi]}$, and different number of points can be extracted from such ellipse through discrete sampling ${ }^{t}$. With regard to sampling point of the ellipse, points with the nearest distance can be selected from the equal-geodesic contour lines as the sampling points for geodesic contour lines. All such sampling points form the post-sampling geodesic contour lines $c_{i} \quad(i=1,2, \ldots, 17)$.

Through carrying out the above-mentioned method, the post-sampling equal-geodesic contour lines shall have the same number of sampling points and the same arrangement order with the corresponding ellipse. Fig. 3 (a) and (b) respectively show the pre-sampling and post-sampling equal-geodesic contour lines.
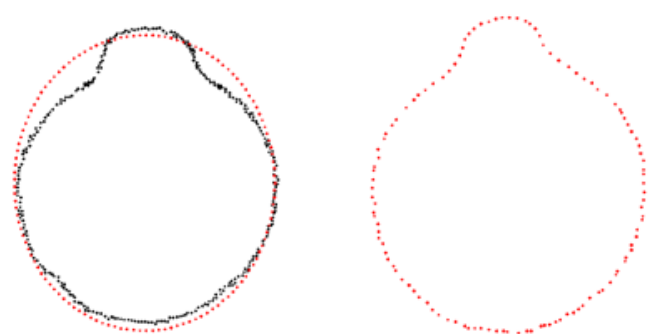

(a) Pre-sampling equal-geodesic contour line (b) Post-sampling equal-geodesic contour line Fig. 3 Sampling of Equal-Geodesic Contour Lines

\section{Local descriptor matching}

Take an arbitrary point $\mathrm{g}\left(\mathrm{g} \in c_{i}\right)$ in the re-sampled equal-geodesic contour line, and draw a sphere with $g$ as the center and $r 1=10 \mathrm{~mm}$ as the radius; face area within the sphere is the Neighborhood L of Point g. Calculate the principal axis of neighborhood point cloud L based on the PCA algorithm, and establish a right-hand coordinate system with the feature vector corresponding to the maximum eigenvalue as the ${ }^{\mathrm{y}}$-axis, feature vector corresponding to the minimum eigenvalue as the $\mathrm{z}$-axis and mass center of point set $o$ as the origin (point). Fig.4 shows the new coordinate system of point cloud L, which is also named as Principal Axis Coordinate System. 


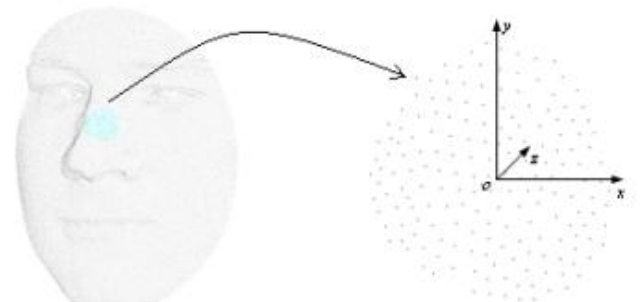

Fig. 4 Principal Axis Coordinate System of Point Set L

Project the points in the point cloud L in three axes of the Principal Axis Coordinate System, and then respectively extract the maximum and minimum projection difference value in three axis directions, namely $f_{x}, f_{y}$ and $f_{z}$. Where, $f_{x}$ and $f_{y}$ respectively reflect the shape distribution of point cloud $\mathrm{L}$ in the $\mathrm{x}$-axis direction and $\mathrm{y}$-axis direction, while $f_{z}$ reflects the curvature of the point set surface; smaller value of $f_{z}$ indicates that the surface $L$ is relatively flat, while greater value of $f_{z}$ indicates that the surface $\mathrm{L}$ is visibly concavo-convex.

\section{Experimental results and analysis}

FRGC v2.0 is a large-scale public face database, in which the 3D face dataset is the largest 3D face database in the world at present, with face data as the 3D point cloud. In this paper, the face data for 41 persons (241 different faces in total) is extracted from the FRGC v2.0 database to carry out the experiment: select the faces with neutral expressions (41 faces in total) to form a library set and the remaining 200 faces (112 faces with neutral expressions and 88 faces with non-neutral expressions) to form the test set.

Representation experiment for local descriptor

To verify the effectiveness of local descriptor in this paper, faces for 3 persons (6 different faces in total) were selected to carry out a comparison. Arbitrarily select a contour line in each face and extract 10 sampling points in the line, and take local features of each sampling point as the y-coordinate. In Fig.5, curves in the same color indicate features of the same person, while curves in different colors show features of different persons. It can be seen from Fig. 5 that local features of the sampling points extracted in this paper have a relatively obvious partition degree for different persons; while for the same person, such partition degree is relatively low. It indicates that local descriptor put forward in this paper has a relatively good performance in representation

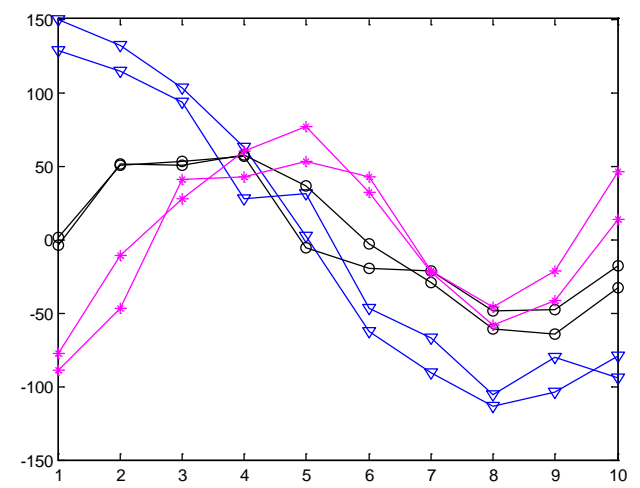

Fig. 5 Representation of Local Descriptor

Face recognition and authentication experiment

From the experiment result, it can be found that, the algorithm can reach the good recognition effect when having no expression, with the recognition rate up to 99.1\%. However, when having expression, the algorithm performance largely decreases, with recognition rate reducing by $9.3 \%$. Analyzing the failing examples with expression, it is found that the most recognition failures occur when the object opens his/her mouth. 


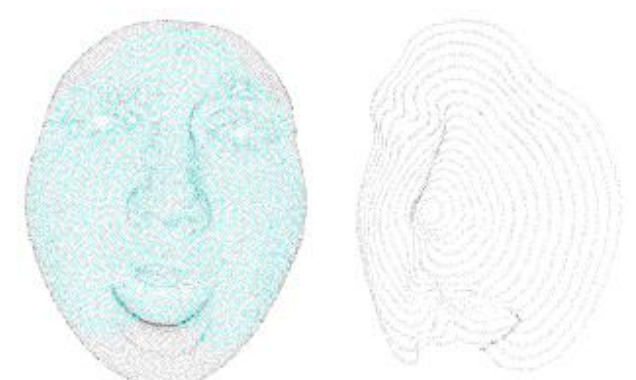

Fig.6 Extracted Equal-geodesic Contour Line When the Mouth Is Opening

Comparing the algorithm of this paper to the literatures [2-5], the result is as shown in Table 1. From the table, it can be seen that, the algorithm of this paper is sufficiently combined with the geometrical information and neighborhood information on the surface of face. Therefore, it shows the certain advantages in the recognition rate and equal error rate.

\begin{tabular}{ccc}
\multicolumn{3}{c}{ Table 1 Comparison of Algorithm Result } \\
\hline Recognition method & \multicolumn{3}{l}{ Recognition rate } & EER \\
\hline Literature [2] & ----- & $5.93 \%$ \\
Literature [3] & $94.67 \%$ & ----- \\
Literature [4] & ----- & $8.32 \%$ \\
Literature [5] & $93.78 \%$ & ----- \\
This paper & $95.5 \%$ & $5.67 \%$ \\
\hline
\end{tabular}

\section{Conclusion}

This paper puts forward a kind of local descriptor to carry out the 3D face recognition. Firstly, the method extracts the face equal-geodesic contour line with re-sampling; then, extract the local descriptor independent of the coordinate information by analyzing the distribution information and area characteristic of local point cloud; finally, carry out the weighted summation rule fusion of local characteristic between corresponding points of the tested face and collected face, and then use the nearest neighbor classifier to obtain the final recognition results. Verifying the performance of algorithm of this paper in the FRGC v2.0 database, the experiment result proves that the method has the good recognition performance. Aiming at that the equal-geodesic contour line is easily to be affected in the bottom half part of face, the improvement measures are raised, and the experiment result proofs the efficiency of the improvement method.

\section{Reference}

[1] Rymbai B, Kandar D, Maji A K. A Hybrid Face Recognition Scheme Using Contour and Gabor Wavelet[M]// Proceedings of International Conference on ICT for Sustainable Development. Springer Singapore, 2016.

[2] Pang Y, Zhang L, Li M, et al. A Novel Gabor-LDA Based Face Recognition Method[M]// Advances in Multimedia Information Processing - PCM 2004. Springer Berlin Heidelberg, 2004:352-358.

[3] Deng Y, Guo Z, Chen Y. Fusing Local Patterns of Gabor and Non-subsampled Contourlet Transform for Face Recognition[C]// Iapr Asian Conference on Pattern Recognition. IEEE Computer Society, 2013:481-485.

[4] Wei X, Yin S, Peng O. A High Precision Feature Based on LBP and Gabor Theory for Face Recognition[J]. Sensors, 2013, 13(4):4499-513.

[5] Sali R, Harsanyi G. Percolation effect in thick film superconductors: Using a $\mathrm{Bi}(\mathrm{Pb}) \mathrm{SrCaCuO}$ based paste to prepare a superconducting planar transformer[J]. 1995. 